Gazi University
Journal of Science
http://dergipark.gov.tr/gujs

\title{
Optimality Conditions of Interval-Valued Optimization Problems by Using Subdifferentials
}

\author{
Emrah KARAMAN \\ Karabük University, Demir Çelik Campus, Faculty of Science, Department of Mathematics, Karabük, Turkey
}

\section{Highlights}

- Interval-valued optimization problems were considered.

- Subdifferential and weak subdifferential were defined for interval-valued functions.

- Some properties of subdifferential and weak subdifferential were examined.

- Necessary and sufficient optimality conditions were obtained for interval-valued optimization problems.

\begin{tabular}{|c|}
\hline Article Info \\
\hline $\begin{array}{l}\text { Received: } 12 / 09 / 2019 \\
\text { Accepted: 03/05/2020 }\end{array}$ \\
\hline Keywords \\
\hline $\begin{array}{l}\text { Optimization, } \\
\text { Subdifferential } \\
\text { Optimality condition } \\
\text { Interval optimization }\end{array}$ \\
\hline
\end{tabular}

\begin{abstract}
In this study, interval-valued optimization problems (shortly, interval optimization) are considered. In order to obtain optimality conditions of interval optimization, subdifferential and weak subdifferential are defined. After some properties including an existence condition of the subdifferentials are studied, optimality conditions including the necessary and sufficient conditions for interval optimization are obtained.
\end{abstract}

\section{INTRODUCTION}

Everybody can meet an optimization problem in life. The goal of the optimization problems is to find the best or worst of the options. When the coefficients of an optimization problems are real numbers, a scalar optimization problem crops out. However, there are uncertain situations as unexpected weather conditions or traffic. Then, these situations can be expressed by closed intervals. In this case, interval-valued optimization problems, which are also called uncertain optimization in some books or papers, arise. Recently, interval optimization has been gathering attention because these optimization problems have applications in engineering, mathematics, control circuitry design, economics, signal processing, global optimization, beam physics, orbits, constraint satisfaction, computer graphics, asteroid, robotics, behavioral ecology, etc. Also, these problems are generalization of scalar optimization problems and they are a special case of set optimization problems. More details about the interval optimization and its applications can be found in the following references.

Methods like derivative, directional derivative, scalarization, vectorization, subdifferential, etc. are used to obtain solutions of optimization problems. Hernández and Rodríguez-Marín [1], Karaman et al. [2] and Xu and $\mathrm{Li}$ [3] obtained some scalarization methods for set optimization. The first vectorization in the optimization theory was used by Küçük et al. [4,5]. Jahn [6] and Karaman et al. [7] obtained some optimality conditions for set optimization by using vectorization. Jahn [8] and Karaman et al. [9] studied on optimality conditions for set optimization by using directional derivative. Hernández and RodríguezMarín [10], Karaman et al. [11] Chen and Jahn [12] obtained some results for set optimization and vector optimization by using subdifferential, respectively. Wu [13] and Bhurjee and Padhan [14] obtained some optimality conditions for interval optimization by using duality. Also, $\mathrm{Wu}$ [15] and Chalco-Cano et al. [16] 
used derivative to obtain solution for interval optimization. But, there is no study to obtain solution of interval optimization by using subdifferential.

There is no natural order relation in order to compare two intervals. Then, some order relations are used to solve the interval optimization problem or compare two intervals. Researchers as Moore [17] and Ishibuchi and Tanaka [18] defined order relations. Since defined order relations are partial order relations, optimal element definitions used for partial order relation are used. Naturally, an efficient element of a family or solution of interval optimization can change when order relation changes.

One of the aim of this study is to obtain some optimality conditions for interval optimization. Subdifferentials are used to achieve this aim. Two subdifferentials are defined and optimality conditions are obtained by using them. In this study, we use the order relation which is given by Moore in [17].

\section{MATERIALS AND METHODS}

In this part, we recall some fundamental definitions which are used in the next section.

Throughout this study, let the class of all bounded and closed intervals (or ranges) in $\mathbb{R}$ be denoted by $\mathbb{I}_{C}$. An interval number in $\mathbb{R}$ is described as follows: For $a_{L} \leq a_{U}$

$$
A=\left[a_{L}, a_{U}\right]=\left\{x \in \mathbb{R}: a_{L} \leq x \leq a_{U}\right\}=\left\{x \in \mathbb{R}:\left\|x-\frac{a_{L}+a_{U}}{2}\right\| \leq \frac{a_{R}-a_{U}}{2}\right\}
$$

where $a_{L}$ and $a_{u}$ are called lower and upper bounds of $A$, respectively. Every real number $x \in \mathbb{R}$ can be considered as a closed and bounded interval as $[x, x]$. Let $A=\left[a_{L}, a_{U}\right]$ and $B=\left[b_{L}, b_{U}\right]$ be intervals in $\mathbb{I}_{C}$ and $k \in \mathbb{R}$. Then, addition, difference of two intervals and scalar multiplication of an interval with scalar $k$ are defined as:

$$
\begin{gathered}
A+B=\left[a_{L}, a_{U}\right]+\left[b_{L}, b_{U}\right]=\left[a_{L}+b_{L}, a_{U}+b_{U}\right], \\
A-B=\left[a_{L}, a_{U}\right]-\left[b_{L}, b_{U}\right]=\left[a_{L}-b_{U}, a_{U}-b_{L}\right]
\end{gathered}
$$

and

$$
k A=k\left[a_{L}, a_{U}\right]= \begin{cases}{\left[k a_{L}, k a_{U}\right]} & \text { if } k \geq 0 \\ {\left[k a_{U}, k a_{L}\right]} & \text { if } k<0\end{cases}
$$

respectively. Also, we have $A-B=A+(-B)$ and $-A=-\left[a_{L}, a_{U}\right]=\left[-a_{U},-a_{L}\right]$ from the difference of two intervals. Moreover, difference of two same intervals may not be zero element.

Let $S$ be a nonempty set. Then, interval-valued function (shortly, interval function) $F: S \rightarrow \mathbb{I}_{C}$ is given by $F(s)=\left[f_{L}(s), f_{U}(s)\right]$ for all $s \in S$ such that $f_{L}(s) \leq f_{U}(s)$. Also, $f_{L}$ and $f_{U}$ are called lower and upper bounded functions, respectively.

Throughout the article, $F: S \rightarrow \mathbb{I}_{C}$ is considered as an interval function and $S \subset \mathbb{R}^{n}(n \geq 1)$. An interval optimization is defined by

$$
(I V O P)\left\{\begin{array}{c}
\min (\max ) F(s) \\
s \in S
\end{array}\right.
$$

In order to obtain the solution and weak solution of $(I V O P)$, we need an order relation on $\mathbb{I}_{C}$. So, we use the following order relations: For $A=\left[a_{L}, a_{U}\right], B=\left[b_{L}, b_{U}\right] \in \mathbb{I}_{C}$

$$
\begin{aligned}
& A \preccurlyeq B \Leftrightarrow a_{U} \leq b_{L}, \\
& A \prec B \Leftrightarrow a_{U}<b_{L} .
\end{aligned}
$$


This order relation is defined by Moore [17]. These order relations are compatible with nonnegative scalar multiplication. But they aren't compatible with addition. For example, although $A \preccurlyeq B, A+C \leqslant B+C$ for $A=[3,4], B=[5,6], C=[-1,3] . \leqslant$ is reflexive, transitive and antisymmetric relation, that is partial order relation. Any two intervals may not be compared according to these order relations because order relation $\preccurlyeq$ is not a total order relation. For example, $A=[3,5]$ and $B=[4,6]$.

Now, we give some basic properties of order relations $\preccurlyeq$ and $\prec$. These properties are used to prove the results in the next section.

Proposition 1. Let $A, B, C, D \in \mathbb{I}_{C}$. Then, the following properties are hold:

(i) $\quad A-B \nless 0 \Leftrightarrow A \$ B \Leftrightarrow-B \nless-A$,

(ii) $\quad A \preccurlyeq B$ and $C \preccurlyeq D \Rightarrow A+C \preccurlyeq B+D$,

(iii) $\quad A \preccurlyeq B \Leftrightarrow A-B \preccurlyeq 0 \Leftrightarrow B-A \nless 0$,

(iv) $\quad A \preccurlyeq B \Rightarrow A-B \preccurlyeq 0$,

(v) $\quad A \prec B \Leftrightarrow A-B<0$,

(vi) $\quad A \nless B \Leftrightarrow A-B \nless 0$.

Proof. The proof can be easily obtained from definitions of $\preccurlyeq$ and $\prec$.

We will use following definition to obtain efficient elements of (IVOP).

Definition 1. Let $F \subset \mathbb{I}_{C}$ and $A \in F$. Then,

(i) $\quad A$ is called a minimal (maximal) interval of $F$ if there isn't any $B \in F$ such that $B \preccurlyeq A(A \preccurlyeq B)$ and $A \neq B$,

(ii) $\quad A$ is called a weak minimal (weak maximal) interval of $F$ if there isn't any $B \in F$ such that $B \prec A(A \prec B)$ and $A \neq B$.

If $F\left(s_{0}\right)$ is a minimal (maximal) interval of $\mathcal{F}(S):=\{F(s): s \in S\}$, then we say that $s_{0}$ is a solution of (IVOP) or $s_{0}$ is a minimal (maximal) solution of (IVOP). Similarly, if $F\left(s_{0}\right)$ is a weak minimal (weak maximal) interval of $\mathcal{F}(S)$, then we say that $s_{0}$ is a weak solution of (IVOP) or $s_{0}$ is called a weak minimal (weak maximal) solution of (IVOP). It is clear that every solution is also a weak solution of the problem. In the rest of the study, the set of all linear operators, which is defined from $\mathbb{R}^{n}$ into $\mathbb{R}$, are denoted by $\mathcal{L}\left(\mathbb{R}^{n}, \mathbb{R}\right)$.

\section{RESULTS AND DISCUSSION}

In this section, two subdifferentials are defined. After some basic properties of subdifferentials are examined, optimality conditions are obtained for (IVOP).

Definition 2. A linear operator $L: \mathbb{R}^{n} \rightarrow \mathbb{R}$ is called a subgradient of $F$ at $s_{0} \in S$ if $F(s)-F\left(s_{0}\right) \prec L(s-$ $s_{0}$ ) for all $s \in S \backslash\left\{s_{0}\right\}$. The set of all subgradients of $F$ at $s_{0}$ is called subdifferential of $F$ at $s_{0}$ and denoted by $\partial F\left(s_{0}\right)$.

Definition 3. A linear operator $L: \mathbb{R}^{n} \rightarrow \mathbb{R}$ is called a weak subgradient of $F$ at $s_{0} \in S$ if $F(s)-F\left(s_{0}\right) \preccurlyeq$ $L\left(s-s_{0}\right)$ for all $s \in S$. The set of all weak subgradients of $F$ at $s_{0}$ is called weak subdifferential of $F$ at $s_{0}$ and denoted by $\partial^{w} F\left(s_{0}\right)$.

It is clear that $\partial F\left(s_{0}\right) \subset \partial^{w} F\left(s_{0}\right)$. The next example shows that the converse implication is not true in general.

Example 1. Let interval function $F:[-1,1] \rightarrow \mathbb{I}_{C}$ be defined as $F(x)=\left[x^{2}, x\right]$ for all $x \in[-1,1]$. Then, we obtain $\partial F(0)=\emptyset$ and $\partial^{w} F(0)=\{1\}$. Although $\partial F(0) \subset \partial^{w} F(0)$, we have $\partial^{w} F(0) \not \subset \partial F(0)$. 
Proposition 2. Let $s_{0} \in K$. Then, $\partial F\left(s_{0}\right)$ and $\partial^{w} F\left(s_{0}\right)$ are convex functions.

Proof. We prove for $\partial F\left(s_{0}\right)$ and second part for $\partial^{w} F\left(s_{0}\right)$ is obtained similarly. Let $L_{1}, L_{2} \in \partial F\left(s_{0}\right)$ and $t \in[0,1]$. Then, we get $F(s)-F\left(s_{0}\right) \prec L_{1}\left(s-s_{0}\right)$ and $F(s)-F\left(s_{0}\right) \prec L_{2}\left(s-s_{0}\right)$ for all $s \in S \backslash\left\{s_{0}\right\}$. Because $\prec$ is compatible with nonnegative scalar multiplication, we have $t F(s)-t F\left(s_{0}\right) \prec t L_{1}\left(s-s_{0}\right)$ and $(1-t) F(s)-(1-t) F\left(s_{0}\right) \prec(1-t) L_{2}\left(s-s_{0}\right)$ for all $s \in S \backslash\left\{s_{0}\right\}$. Then, we get $t F(s)-t F\left(s_{0}\right)+$ $(1-t) F(s)-(1-t) F\left(s_{0}\right) \prec t L_{1}\left(s-s_{0}\right)+(1-t) L_{2}\left(s-s_{0}\right)$ from Proposition 1 (ii). This gives $F(s)-F\left(s_{0}\right)<t L_{1}\left(s-s_{0}\right)+(1-t) L_{2}\left(s-s_{0}\right)=\left(t L_{1}+(1-t) L_{2}\right)\left(s-s_{0}\right)$ for all $s \in S \backslash\left\{s_{0}\right\}$. Hence, $t L_{1}+(1-t) L_{2} \in \partial F\left(s_{0}\right)$ and $\partial F\left(s_{0}\right)$ is convex function.

Proposition 3. Let $t \in \mathbb{R}_{+}$. Then, $\partial(t F)\left(s_{0}\right)=t \partial F\left(s_{0}\right)$ for all $x_{0} \in S$.

Proof. Since the order relation $\preccurlyeq$ is compatible with nonnegative scalar multiplication, we get

$$
\begin{aligned}
t \partial F\left(s_{0}\right) & =t\left\{L \in \mathcal{L}\left(\mathbb{R}^{n}, \mathbb{R}\right): F(s)-F\left(s_{0}\right) \prec L\left(s-s_{0}\right), s \in S \backslash\left\{s_{0}\right\}\right\} \\
& =\left\{t L \in \mathcal{L}\left(\mathbb{R}^{n}, \mathbb{R}\right): F(s)-F\left(s_{0}\right) \prec L\left(s-s_{0}\right), s \in S \backslash\left\{s_{0}\right\}\right\} \\
& =\left\{L \in \mathcal{L}\left(\mathbb{R}^{n}, \mathbb{R}\right): F(s)-F\left(s_{0}\right) \prec \frac{L}{t}\left(s-s_{0}\right), s \in S \backslash\left\{s_{0}\right\}\right\} \\
& =\left\{L \in \mathcal{L}\left(\mathbb{R}^{n}, \mathbb{R}\right): t F(s)-t F\left(s_{0}\right) \prec L\left(s-s_{0}\right), s \in S \backslash\left\{s_{0}\right\}\right\} \\
& =\partial(t F)\left(s_{0}\right) .
\end{aligned}
$$

Proposition 4. Let $s_{0} \in S$. Then, the set $\partial F\left(s_{0}\right)$ is closed for all $s_{0} \in S$.

Proof. If $\partial F\left(s_{0}\right)=\emptyset$, then proof is completed. Assume that $\partial F\left(s_{0}\right) \neq \varnothing$ and $L \in \operatorname{cl}\left(\partial F\left(s_{0}\right)\right)$. We show $L \in \partial F\left(s_{0}\right)$.

Suppose to the contrary that $L \notin \partial F\left(s_{0}\right)$. Then, there exists a $s_{0} \in S$ such that $F(s)-F\left(s_{0}\right) \nless L\left(s-s_{0}\right)$. Since $L \in \operatorname{cl}\left(\partial F\left(s_{0}\right)\right)$ there exists a sequence $L_{n} \in \partial F\left(s_{0}\right)$ such that $L_{n} \rightarrow L$. Because $L_{n} \in \partial F\left(s_{0}\right)$ for all $n \in \mathbb{N}$, we have

$$
F(s)-F\left(s_{0}\right) \prec L_{n}\left(s-s_{0}\right) \text { for all } x \in S \backslash\left\{s_{0}\right\} .
$$

As interval $L_{n}\left(s-s_{0}\right)-F(s)+F\left(s_{0}\right)$ is closed and bounded set, we get $F(s)-F\left(s_{0}\right) \prec L\left(s-s_{0}\right)$ for $n \rightarrow \infty$. Hence, $L \in \partial F\left(s_{0}\right)$. This is a contradiction. Therefore, $\partial F\left(s_{0}\right)$ is closed.

Proposition 5. Let $F, G: X \rightarrow \mathbb{I}_{C}$ be interval functions and $s_{0} \in S$. Then, $\partial F\left(s_{0}\right)+\partial G\left(s_{0}\right) \subset$ $\partial(F+G)\left(s_{0}\right)$.

Proof. Let $L_{1} \in \partial F\left(s_{0}\right)$ and $L_{2} \in \partial G\left(s_{0}\right)$. Then, we have $F(s)-F\left(s_{0}\right) \prec L_{1}\left(s-s_{0}\right)$ and $G(s)-G\left(s_{0}\right) \prec$ $L_{2}\left(s-s_{0}\right)$ for all $s \in S \backslash\left\{s_{0}\right\}$. From Proposition 1 (ii), we have $F(s)-F\left(s_{0}\right)+G(s)-G\left(s_{0}\right) \prec$ $L_{1}\left(s-s_{0}\right)+L_{2}\left(s-s_{0}\right)$, i.e. $(F+G)(s)-(F+G)\left(s_{0}\right) \prec\left(L_{1}+L_{2}\right)\left(s-s_{0}\right)$. Then, $L_{1}+L_{2} \in \partial(F+$ $G)\left(s_{0}\right)$, i.e. $\partial F\left(s_{0}\right)+\partial G\left(s_{0}\right) \subset \partial(F+G)\left(s_{0}\right)$.

Now, we give an existence condition of subdifferential of an interval function.

Proposition 6. Let $s_{0} \in S$. If there exist a constant $C$ such that

$$
C\left|s-s_{0}\right| \subsetneq F(s)-F\left(s_{0}\right)
$$

for all $s \in S \backslash\left\{s_{0}\right\}$, then $\partial F\left(s_{0}\right) \neq \varnothing$.

Proof. Assume that there is a constant $C$ such that $C\left|s-s_{0}\right| \subsetneq F(s)-F\left(s_{0}\right)$ for all $s \in S \backslash\left\{s_{0}\right\}$. Then, we have $F(s)-F\left(s_{0}\right) \prec C\left|s-s_{0}\right|$ for all $s \in S \backslash\left\{s_{0}\right\}$. Hence, we yield $C \in \partial F\left(s_{0}\right) \neq \varnothing$ from definition of subdifferential. 
In the rest of the study, we will give the optimality conditions for (IVOP).

Theorem 1. Let $s_{0} \in S$. $s_{0}$ is a minimal solution of (IVOP) if and only if $0 \notin \partial F\left(s_{0}\right)$.

Proof. Assume that $s_{0}$ is a minimal solution of (IVOP). Then, we have $F(s) \$ F\left(s_{0}\right)$ for all $s \in S \backslash\left\{s_{0}\right\}$. By Proposition 1 (iv), we get $F(s)-F\left(s_{0}\right) \nless 0$ for all $s \in S \backslash\left\{s_{0}\right\}$. So, $0 \notin \partial F\left(s_{0}\right)$.

The other direction can be obtained similarly.

Theorem 2. Let $s_{0} \in S$. If $0 \in \partial F\left(s_{0}\right)$, then $s_{0}$ is a maximal solution of (IVOP).

Proof. Let $0 \in \partial F\left(s_{0}\right)$. Then $F(s)-F\left(s_{0}\right) \prec 0$ for all $s \in S \backslash\left\{s_{0}\right\}$. From Proposition $1(\mathrm{v})$, we get $F(s) \prec$ $F\left(s_{0}\right)$ for all $s \in S \backslash\left\{s_{0}\right\}$. Hence, $F(s) \leqslant F\left(s_{0}\right)$ for all $s \in S \backslash\left\{s_{0}\right\}$ and $s_{0}$ is a maximal solution of $(I V O P)$.

Corollary 1. Let $s_{0} \in S$. If $0 \in \partial F\left(s_{0}\right)$, then $s_{0}$ is a weak maximal solution of (IVOP).

Theorem 3. Let $s_{0} \in S$. If $0 \in \partial^{w} F\left(s_{0}\right)$, then $s_{0}$ is a maximal solution of (IVOP).

Proof. The proof can be obtained similar to Theorem 2 by using Proposition 1 (iii).

Example 2. Let interval function $F:[-1,1] \rightarrow \mathbb{I}_{C}$ be defined as $F(x)=\left[x^{2},|x|\right]$ for all $x \in[-1,1]$. Consider the following interval optimization

$$
(I V O P)\left\{\begin{array}{c}
\min (\max ) F(x) \\
x \in[-1,1]
\end{array}\right.
$$

Let's calculate subdifferential and weak subdifferential of $F$ at 0 and 1.

$$
\begin{aligned}
\partial F(0) & =\{L: \mathbb{R} \rightarrow \mathbb{R}: F(x)-F(0) \prec L(x), x \in[-1,1] \backslash\{0\}\} \\
& =\{t \in \mathbb{R}: F(x) \prec t x, x \in[-1,1] \backslash\{0\}\} \\
& =\left\{t \in \mathbb{R}:\left[x^{2},|x|\right] \prec t x, x \in[-1,1] \backslash\{0\}\right\} \\
& =\{t \in \mathbb{R}:|x|<t x, x \in[-1,1] \backslash\{0\}\} \\
& =\emptyset
\end{aligned}
$$

Since $0 \notin \partial F(0), 0$ is a minimal solution of (IVOP) from Theorem 1 .

$$
\begin{aligned}
\partial^{w} F(0) & =\{L: \mathbb{R} \rightarrow \mathbb{R}: F(x)-F(0) \preccurlyeq L(x), x \in[-1,1]\} \\
& =\{t \in \mathbb{R}: F(x) \preccurlyeq t x, x \in[-1,1]\} \\
& =\left\{t \in \mathbb{R}:\left[x^{2},|x|\right] \preccurlyeq t x, x \in[-1,1]\right\} \\
& =\{t \in \mathbb{R}:|x| \leq t x, x \in[-1,1]\} \\
& =\emptyset . \\
\partial F(1) & =\{L: \mathbb{R} \rightarrow \mathbb{R}: F(x)-F(1) \prec L(x-1), x \in[-1,1)\} \\
& =\{t \in \mathbb{R}: F(x)-F(1) \prec t(x-1), x \in[-1,1)\} \\
& =\left\{t \in \mathbb{R}:\left[x^{2},|x|\right]-[1,1] \prec t(x-1), x \in[-1,1)\right\} \\
& =\left\{t \in \mathbb{R}:\left[x^{2}-1,|x|-1\right] \prec t(x-1), x \in[-1,1)\right\} \\
& =\{t \in \mathbb{R}:|x|-1<t(x-1), x \in[-1,1)\} \\
& =(-\infty, 1) .
\end{aligned}
$$

Since $0 \in \partial F(1), 1$ is a maximal solution of (IVOP) from Theorem 2.

$$
\begin{aligned}
\partial^{w} F(1) & =\{L: \mathbb{R} \rightarrow \mathbb{R}: F(x)-F(1) \preccurlyeq L(x-1), x \in[-1,1]\} \\
& =\{t \in \mathbb{R}: F(x)-F(1) \preccurlyeq t(x-1), x \in[-1,1]\} \\
& =\left\{t \in \mathbb{R}:\left[x^{2},|x|\right]-[1,1] \preccurlyeq t(x-1), x \in[-1,1]\right\}
\end{aligned}
$$




$$
\begin{aligned}
& =\{t \in \mathbb{R}:|x|-1 \leq t(x-1), x \in[-1,1]\} \\
& =(-\infty, 0] .
\end{aligned}
$$

1 is a weak maximal solution of (IVOP) from Theorem 3 since $0 \in \partial^{w} F(1)$.

\section{CONCLUSION}

In this paper, two subdifferentials are considered for interval optimization. Some basic properties including an existence condition are obtained. Also, optimality conditions are derived for corresponding problem. Results are explained by using examples. This method can be applied to other order relations on interval optimization. Some new methods as used in vector or set optimization can be improved. Moreover, this method can also be applied to the optimal control problems in $[19,20]$.

\section{CONFLICT OF INTEREST}

No conflict of interest was declared by the author.

\section{REFERENCES}

[1] Hernandez, E., Rodriguez-Marin, L., "Nonconvex scalarization in set optimization with set-valued maps", J. Math. Anal. Appl., 325: 1-18, (2007).

[2] Karaman, E., Soyertem, M., Atasever Güvenç, İ., Tozkan, D., Küçük, M., Küçük, Y., "Partial order relations on family of sets and scalarizations for set optimization", Positivity, 22(3): 783-802, (2018).

[3] Xu Y. D., Li S. J., “A new nonlinear scalarization function and applications”, Optim., 65: 207-231, (2016).

[4] Küçük, M., Soyertem, M., Küçük, Y., "The generalization of total ordering cones and vectorization to separable Hilbert spaces”, J. Math. Anal. Appl., 389: 1344-1351, (2012).

[5] Küçük, M., Soyertem, M., Küçük, Y., Atasever, İ., "Vectorization of set-valued maps with respect to total ordering cones and its applications to set-valued optimization problems", J. Math. Anal. Appl., 385: 285-292, (2012).

[6] Jahn, J., "Vectorization in set optimization", J. Optimiz. Theory. App., 167: 783-795, (2013).

[7] Karaman, E., Soyertem, M., Atasever Güvenç, İ., Tozkan, D., Küçük, M., Küçük, Y., “A Vectorization for nonconvex set-valued optimization," Turk. J. Math., 42: 1815-1832, (2018).

[8] Jahn, J., "Directional derivatives in set optimization with the set less order relation" Taiwan. J. Math. 19: 737-757, (2015).

[9] Karaman, E., Soyertem, M., Atasever Güvenç, İ., "Optimality conditions in set-valued optimization problem with respect to a partial order relation via directional derivative", Taiwan. J. Math., (2019). doi: $10.11650 / \mathrm{tjm} / 190604$.

[10] Hernández, E., Rodríguez-Marín, L., "Weak and strong subgradients of set-valued maps", J. Optim. Theory. Appl., 149: 352-365, (2011).

[11] Karaman, E., Atasever Güvenç, İ., Soyertem, M., "Optimality conditions in set-valued optimization problems with respect to a partial order relation by using subdifferentials", Optimization, (2020). doi: 10.1080/02331934.2020.1728270. 
[12] Chen, G.Y., Jahn, J., "Optimality conditions for set-valued optimization problems”, Math. Methods. Oper. Res., 48 (2): 187-200, (1998).

[13] Wu, H.C., "Duality Theory for Optimization Problems with Interval-Valued Objective Functions", J. Optim. Theory. Appl., 144: 615-628, (2010).

[14] Bhurjee, A. K., Padhan, S. K., "Optimality conditions and duality results for non-differentiable interval optimization problems", J. Appl. Math. Comput., 50: 59-71, (2016).

[15] Wu, H.C., "The Karush-Kuhn-Tucker optimality conditions in an optimization problem with intervalvalued objective function”, Eur. J. Oper. Res., 176: 46-59, (2007).

[16] Chalco-Cano, Y., Lodwick, W. A., Rufian-Lizana, A., "Optimality conditions of type KKT for optimization problem with interval-valued objective function via generalized derivative", Fuzzy. Optim. Decis. Making., 12: 305-322, (2013).

[17] Moore, R.E., Method and Application of Interval Analysis, SIAM, Philadelphia, (1979).

[18] Ishibuchi, H., Tanaka, H., "Multiobjective programming in optimization of the interval objective function”, Eur. J. Oper. Res., 48(2): 219-225, (1990).

[19] Çıbık, A. B., Yılmaz, F. N., "Variational multiscale method for the optimal control problems of convection-diffusion-reaction equations", Turk. J. Math., 42: 164-180, (2018).

[20] Çıbık, A., "The effect of a sparse grad-div stabilization on control of stationary Navier-Stokes equations", J. Math. Anal. Appl., 437: 613-628, (2016). 